\title{
Lensed Arcs and Inner Structure of Abell $697^{1}$
}

\author{
Mark R. Metzger ${ }^{2}$ and Chung-Pei $\mathrm{Ma}^{3}$
}

\begin{abstract}
We present new optical observations of the $z=0.282$ cluster Abell 697 from the Keck II telescope. Images show an unusual disturbed structure in the $\mathrm{cD}$ halo and a previously unknown faint gravitational lens arc. A spectrum of the arc did not yield a redshift, but its spectrum and colors suggest it lies at $z>1.3$. We construct models to reproduce the arc that show the potential is likely to be highly elliptical. We suggest that this cluster may have undergone a recent merger and is in the process of forming its $\mathrm{cD}$ galaxy. Analysis of X-ray data from ROSAT and ASCA suggests that the merging process is sufficiently advanced that the gas in the cluster has relaxed, and A697 lies near the $L_{x}-T_{x}$ relation for normal clusters.
\end{abstract}

Subject headings: galaxies: clusters: individual (Abell 697) — galaxies: elliptical and lenticular, $\mathrm{cD}$ - gravitational lensing

\section{Introduction}

Clusters of galaxies represent the largest scales in the universe at which gravitational collapse has become non-linear and virialization takes place. As such, they also are the warehouses of most of the visible baryons in the universe: hydrogen gas that falls into the cluster and heats in the process, forming an intracluster medium too hot to be bound by individual galaxies. Massive clusters therefore also tend to be strong sources of X-ray emission via thermal bremsstrahlung of the intracluster gas, which provides a means for clusters to be identified in addition to the overdensity of visible galaxies.

\footnotetext{
${ }^{1}$ Based on observations obtained at the W.M. Keck Observatory, operated as a scientific partnership by the California Institute of Technology, the University of California, and the National Aeronautics and Space Administration.

${ }^{2}$ Division of Physics, Mathematics, and Astronomy, California Institute of Technology, MS 105-24, Pasadena, CA 91125; mrm@caltech.edu

${ }^{3}$ Department of Physics and Astronomy, University of Pennsylvania, Philadelphia, PA 19104; cpma@strad.physics. upenn.edu
} 
Clusters also serve as cosmological probes, as their formation and evolution rate depend on cosmological parameters and the kinematics of the dark matter. While optical surveys have led to the discovery of most clusters at low redshift, most of the known high-redshift clusters have been discovered through X-ray surveys (e.g. the EMSS survey, Gioia et al. 1990). To interpret cluster evolution from high redshift to the present requires a proper understanding of the differences between X-ray and optical cluster samples, each with their advantages and pitfalls. Abell 697 (at $z=0.282$ ) is a cluster that represents an overlap between the two samples. Discovered optically (Abell et al. 1989), it is fairly rich and is one of the higher redshift clusters in the ACO catalog. It is also X-ray luminous and has sufficient flux to be included in X-ray selected cluster samples (Ebeling et al. 1998).

We report new Keck imaging and spectroscopic observations of the central $6^{\prime}$ of A697. The images show a highly asymmetric cD halo around A697, with low surface brightness components that may be remnants of recent merger activity. Our imaging also reveals a faint, blue, arc-like feature which is likely to be lensed by the cluster core. Deep long-slit spectroscopy of the arc did not yield a direct redshift for this object, but does provide several cluster galaxy redshifts to estimate a velocity dispersion for the cluster. The combination of the blue color of the arc and the lack of strong emission lines in the optical spectrum suggest that the arc is at $z \gtrsim 1.3$. We construct a simple model of the cluster potential near the core to explain the lensed feature, and suggest two possibilities for the more extended, arc-like red structure found to the northwest of the core.

\section{Observations and Data Reduction}

Images of the central region of A697 were obtained on UT January 28, 1998 on the Keck II $10 \mathrm{~m}$ telescope with LRIS (Oke et al. 1995). The optics illuminate a $6^{\prime} \times 8^{\prime}$ field onto a $2048 \times 2048 \mathrm{CCD}$ with pixels projecting to $0^{\prime \prime} .211$ square on the sky. Multiple dithered images were obtained in each of the $V, R$, and $I$ bands, which were debiased, flatfield-corrected with twilight sky illumination, and corrected for gain variation. The

images were then registered and combined using the "Mora" reduction package (Metzger 1994); bad pixels were removed from the stack, and the final image was constructed using a weighted sum. The total exposure time was $3000 \mathrm{~s}$ in $V$ and $R$, and $2700 \mathrm{~s}$ in I. Photometric calibration was provided by observing standard fields of Landolt (1992). Figure 1 shows a color image of the inner $4^{\prime}$ of the cluster.

A quick reduction of the images at the telescope revealed a blue arc-like structure to the southwest of the $\mathrm{cD}$. We proceeded to obtain three dithered $1200 \mathrm{~s}$ longslit spectra of the arc with LRIS using a $300 \ell \mathrm{mm}^{-1}$ grating on UT January 30, 1999, at a sky position angle of $83 .{ }^{\circ} 8$. The individual frames were processed with Mora to remove the amplifier bias and nonlinearity, and to correct the response with internal halogen lamp spectra. 
The three frames were aligned and co-added, rejecting cosmic rays using an iterative filter. Wavelength calibration was obtained using $\mathrm{Hg}-\mathrm{Kr}-\mathrm{Ne}-\mathrm{Ar}$ lamp spectra, and the arc spectrum was extracted from the 2-d frame by adding flux over the region where the arc was visible on the image and subtracting sky from adjacent uncontaminated regions on the slit. Flux calibration was obtained using a spectrum of Feige 67 (Oke 1990). No significant emission or absorption lines are visible in the arc spectrum. When averaged over $500 \AA$ bins, we find only a marginal continuum detection from the arc, but do not have enough signal-to-noise to obtain an absorption redshift via cross-correlation or template matching.

An additional $1200 \mathrm{~s}$ spectrum was taken centered on the bright cD nucleus (see Fig. 2) and aligned with the secondary nucleus at a sky PA of $28^{\circ}$. The two long-slit spectra also capture a number of nearby galaxies in the field, which are included in our sample to estimate of the cluster velocity dispersion. For the galaxies without strong emission lines, the well-exposed spectrum of the main $\mathrm{cD}$ nucleus was used as a cross-correlation template; we measure $z=0.2824$ from the $\mathrm{Ca}$ II and $\mathrm{H} \beta$ absorption features, in good agreement with the value measured by Crawford et al. (1995). The velocities for 11 galaxies relative to the cD are given in Table 1; 9 of these can be considered members of A697 based on proximity in redshift space. Using all 9 galaxies, we derive a velocity dispersion of $\sigma=941 \pm 296$ $\mathrm{km} \mathrm{s}^{-1}$ (rest frame). However, the distribution of the velocities is non-Gaussian: two of the galaxies lie at relatively large velocities from the main $\mathrm{cD}$ nucleus, at $-2735 \mathrm{~km} \mathrm{~s}^{-1}$ and $-2044 \mathrm{~km} \mathrm{~s}^{-1}$. Removing these reduces $\sigma$ to $553 \mathrm{~km} \mathrm{~s}^{-1}$ from 7 galaxies, at a mean velocity relative to the $\mathrm{cD}$ of $93 \pm 85 \mathrm{~km} \mathrm{~s}^{-1}$. The possibility of the presence of subgroups dictates that more velocities are needed to obtain a reliable dispersion, though our two estimates probably give reasonable bounds. The lower dispersion, however, would be unusually low for a cluster with such a high intracluster gas temperature and X-ray luminosity (see $\S$ 3).

We also attempted to measure dispersion within the $\mathrm{cD}$ halo itself as a function of radius, but significant contamination from interloping galaxies prevented an accurate

estimate by artificially enhancing the apparent line widths. Our cD spectrum also shows no sign of $\mathrm{H} \alpha$ emission to an upper limit of $W_{\lambda}(\mathrm{obs}) \leq 0.5 \AA$, in contrast to the Crawford et al. (1995) measurement of $3.2 \pm 1.4 \AA$.

\section{Cluster Properties}

The central region of the cluster is dominated by a bright elliptical galaxy with a cD halo profile (Fig. 1), having total V magnitude of 18.1. A secondary nucleus is also present $2^{\prime \prime}$ to the northeast. At a radius of $5^{\prime \prime}$, the $\mathrm{cD}$ halo has a roughly elliptical profile with axis ratio $b / a=0.78$, but at larger radius the halo becomes significantly asymmetric with a large extension visible to $r=40^{\prime \prime}$ to the southeast at surface brightness of $\mathrm{V}=28 \mathrm{mag}$ $\operatorname{arcsec}^{-2}$. The orientation of the inner $\mathrm{cD}$ isophotes, at roughly $\mathrm{PA}=163^{\circ}$, is well aligned 
with the ASCA 2-10 keV image brightness contours at larger radius (see below).

An unusual, low surface brightness extended feature is seen to the northwest of the cD center. The surface brightness varies irregularly from 24.5 to $25.5 \mathrm{~V}$ mag $\operatorname{arcsec}^{-2}$ over the central region covering about $100 \operatorname{arcsec}^{2}$. The color of much of the extended source is consistent with that of an old stellar population at this redshift (as well as the cluster ellipticals), suggesting it is comprised of stars and is possibly a remnant of tidally disrupted galaxies. Part of the source extends in the direction of one of the cluster ellipticals, but superposed on this wide extension is a more narrow, bluer arc-like structure that may be an image of a lensed background galaxy (see $\S$ 田).

X-ray observations of A697 with ROSAT and ASCA were obtained from the NASA HEASARC public data archive. A697 appears near the edge of the field of a $10800 \mathrm{sec}$ ROSAT PSPC observation of GBS 0839+37, and is cataloged in the ROSAT Bright Cluster Survey (Ebeling et al. 1998) with total luminosity of $1.6 \times 10^{45} h_{50}^{-2} \mathrm{erg} \mathrm{s}^{-1}$ in the energy range from $0.1-2 \mathrm{keV}$ (observed frame). The data gives a wide field of view to the north and west of the cluster center, showing no other hot clusters nearby that might be part of an aligned large-scale structure. A $28000 \mathrm{HRI}$ image centered on the field likewise shows no bright extended structures to the southeast. The ASCA data from both GIS and SIS were reduced and analyzed using the XSPEC software package (Arnaud 1996). A fit to the spectrum using a Raymond-Smith model as implemented in XSPEC yields a temperature of $10.2 \pm 1.9 \mathrm{keV}$, which lies fairly close to the Lx-Tx relation (Mushotzky \& Scharf 1998). The data also reveal an extended x-ray source to the southwest (off of our optical images), possibly associated with an additional infalling group. Ellipses fit to the X-ray isophotes at $6^{\prime}$ major axis diameter give an axis ratio of $b / a=0.68$ oriented at $\mathrm{PA}=155^{\circ}$.

\section{Lens Models}

We use an elliptical potential of the form

$$
\psi\left(r^{\prime}\right)=4 \pi\left(\frac{\sigma_{1 D}}{c}\right)^{2} \frac{D_{L S}}{D_{S}}\left(r_{c}^{2}+r^{\prime 2}\right)^{\frac{1}{2}}
$$

to model the cluster as a lens (Blandford \& Kochanek 1987). The variable $\sigma_{1 D}$ is the line-ofsight velocity dispersion in the limit $r^{\prime} \gg r_{c}$ for the spherical case, $D_{S}$ and $D_{L S}$ are angular size distances to the source and from the lens to the source, respectively, and $r_{c}$ defines a softening radius. The ellipticity enters in the definition of $r^{\prime}, r^{\prime 2}=\left(1-\epsilon_{p}\right) x^{2}+\left(1+\epsilon_{p}\right) y^{2}$, where the coordinates $x, y$ are aligned along the major and minor axes of the potential (see Mellier et al. 1993 for details about $\epsilon_{p}$ ). This represents a softened isothermal sphere for $\epsilon_{p}=0$, and models the shape of dark halo potentials fairly well near the cores of clusters (e.g., Tyson et al. 1998). We choose the more limited relation with a fixed power law, as there are few lensing constraints in this system to differentiate various potential profiles. 
We search for the best-fit model by minimizing the distances between the predicted image positions and the observed arc positions with respect to the free model parameters. In each iteration, the observed arc pixels are first mapped onto the source plane using the current model parameters. We then find all the image locations of each source with a grid searching algorithm that maps a fine grid in the lens plane to the source plane and then scans for pixels in the lens plane whose mapped pixels coincide with the source positions.

Although few observational constraints are available in this system, the unusual orientation of the southern arc-like object makes this lens nontrivial to model. Its center of curvature points about $5^{\prime \prime}$ away (toward the west) from the cD center, and this feature cannot be modeled by a single potential centered at the $\mathrm{cD}$ with the measured orientation $\theta=73^{\circ}$ (i.e. $\mathrm{PA}=163^{\circ}$ ) and potential ellipticity $\epsilon_{p}=0.08$ that is suggested by the $\mathrm{cD}$ profile. Given the disturbed surface brightness distribution surrounding the $\mathrm{cD}$, however, it is conceivable that the shape of the cluster potential does not trace the $\mathrm{cD}$ halo exactly. Once $\theta$ and $\epsilon_{p}$ for the cluster are allowed to vary, we are able to construct a simple model that reasonably reproduces the position and shape of the southern arc (see Fig. (3)). The potential for the cluster in this model is centered at the $\mathrm{cD}$ and has a velocity dispersion $\sigma_{1 D}=910 \mathrm{~km} \mathrm{~s}^{-1}$, core radius $r_{c}=10^{\prime \prime}$, ellipticity $\epsilon_{p}=0.22$, and orientation $\theta=55^{\circ}$. The source is placed at $z_{s}=1.4$, consistent with the lower limit of 1.3 from our spectrum. An additional singular elliptical potential with $\sigma_{1 D}=200 \mathrm{~km} \mathrm{~s}^{-1}$ and the measured cD profile is used to represent the $\mathrm{cD}$ galaxy. As Figure 3 shows, the southern arc is a fold arc, and the counter-image appears east of the $\mathrm{cD}$. Our ground-based image shows a number of faint blue, arclike candidates in this area. High resolution HST data will be needed for more detailed morphological information and more refined modeling. Due to the lack of arclike objects in the area for possible 4th and 5th images, we favor a three image lens model (here two merge to form an arc). The ellipticity of 0.22 helps to flatten the contour of the radial caustic shown in Figure 3 and allows the source to be placed outside of it while remaining near a fold of the tangential caustic.

The northern arc-like object can also be reproduced in our lens model. The lack of a counterarc suggests that the simplest way to accommodate this candidate arc is for it to be a single distorted image of a red galaxy, which can occur if the source is outside of both caustics and the arc is outside of both critical curves. This scenario would require the source redshift to be below 1.4, given that the tangential critical curve for the source of the

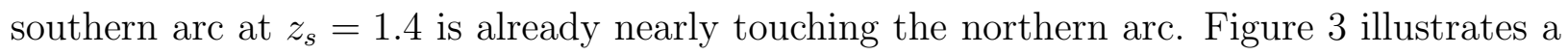
plausible source position for the northern object assuming $z_{s}=1.2$. 


\section{Discussion and Summary}

Together, the asymmetric cD profile, extended features, and highly elliptical potential implied by the arc model suggest that A697 may have recently undergone a significant merger event (e.g. Roettiger et al. 1996). In this scenario, the ellipticity and more highly extended outer contours are a dynamical consequence of the merger, in which the tidal tails produced in the encounters have not had time to relax. A more highly elliptical potential could result from two massive dark matter cores that have not completed the merger. Several observational consequences to the intracluster gas would also be predicted in this case, including regions of non-uniform, elevated temperature and X-ray luminosity (Evrard, Metzler, \& Navarro 1996; Ricker 1998; Jones et al. 1997; Churazov et al. 1999).

Another scenario that could explain the complex structure is that the $\mathrm{cD}$ is undergoing the process of forming its extended halo, possibly from the merging of several massive galaxies. The dark matter simulations made by Dubinski (1998) suggest that this scenario can produce massive galaxies similar in appearance to a cD. His figure 3 shows a $\mathrm{cD}$ in the process of formation, and several features including tidal stripping and halo asymmetry qualitatively agree with our observations. However, many of the galaxies near the cluster core appear tidally disrupted, while few of the concentrated ellipticals show this effect in A697. Given that these simulations did not include intracluster gas and were of finite resolution, though, it is difficult to draw any quantitative conclusions (though it is suggestive). The significant disruption of the $\mathrm{cD}$ halo and the presence of the low surface brightness source, interpreted as a formation event, provides some evidence in favor of the major-merger scenario for $\mathrm{cD}$ halo formation over more gentle processes (e.g. Lopez-Cruz et al. 1997), but of course may not be the only formation mechanism.

The detailed X-ray structure of A697 will be an interesting probe into the physics of this merger. Simple models may not fit, as recent mergers may leave shocks in gas producing a complex inner temperature and brightness structure. More detailed models may help to confirm a recent merger, as some of these structures should dissipate rapidly, and specific emission lines from the X-ray gas should be measurable with new instruments aboard XMM and Chandra. Such a model of the merging inner potential would also benefit from deep HST images of the core, from which additional faint arcs or arclets could provide further model constraints and resolution.

Our deep optical imaging of A697 reveals several arc-like objects and a complex inner structure. A spectrum of the lower arc did not display obvious emission lines; combined with its blue color, this indicates $z>1.3$ for the lensed galaxy. We have constructed a potential model to reproduce the arc's tilted orientation. The model agrees to within the rather large uncertainties of the velocity dispersion that was estimated from nine galaxy spectra. A possible arc on the northern side of the cluster can also be reproduced in this 
model. The $\mathrm{cD}$ itself, while having a normal spectrum, has an unusual and highly disturbed halo profile, including possible remnants of recently disrupted old cluster galaxies. The potential model implied by the arc structure is also highly elliptical, and we suggest that this is due to a recent merger event, either between subcluster elements or massive galaxies acting to form the $\mathrm{cD}$. We also suggest that this cluster would provide an ideal system for testing numerical simulation predictions of the effects of cluster merging on the intracluster gas with high-resolution images and spectra in optical and X-ray wavelengths.

We thank Roger Blandford, Una Hwang, and John Blakeslee for helpful discussions, and Terry Stickel and Ron Quick for assistance at the telescope. This research has made use of NED, operated by the Jet Propulsion Laboratory, Caltech, under contract with NASA, and data obtained from the HEASARC online service, provided by NASA's Goddard Space Flight Center. M.R.M. acknowledges support from Caltech, NASA grant AR-07520.01-96A, and NSF grant AST 9820664. C.-P. M. acknowledges support of an Alfred P. Sloan Foundation Fellowship, a Cottrell Scholars Award from the Research Corporation, a Penn Research Foundation Award, and NSF grant AST 9973461. 


\section{REFERENCES}

Abell, G. O., Corwin H. G. Jr. \& Olowin, R. P. 1989, ApJS, 70, 1

Arnaud, K.A., 1996, in Astronomical Data Analysis Software and Systems V, eds. G. Jacoby \& J. Barnes, ASP Conf. Series volume 101, 17

Blandford, R. D. \& Kochanek, C. S. 1987, ApJ, 321, 658

Churazov, E., Gilfanov, M., Forman, W., \& Jones, C. 1999, ApJ 520, 105

Crawford, C. S., Edge, A. C., Fabian, A. C., Allen, S. W., Bohringer, H., Ebeling, H., McMahon, R. G., \& Voges, W. 1995, MNRAS 274, 75

Dubinski, J. 1998, ApJ, 502, 141

Ebeling, H., Edge, A. C., Bohringer, H., Allen, S. W., Crawford, C. S., Fabian, A. C., Voges, W., Huchra, J. P. 1998, MNRAS, 301, 881

Evrard, A. E., Metzler, C. A., \& Navarro, J. F. 1996, ApJ, 469, 494

Gioia, I. M., Maccacaro, T., Schild, R. E., Wolter, A., Stocke, J. T., Morris, S. L., \& Henry, J. P. 1990, ApJS, 72, 567

Landolt, A. U. 1992, AJ, 104, 340

Lopez-Cruz, O., Yee, H. K. C., Brown, J. P., Jones, C., \& Forman, W. 1997, ApJL, 475, 97.

Mellier, Y., Fort, B., \& Kneib, J.-P. 1993, ApJ, 407, 33

Metzger, M. R. 1994, Ph.D. Thesis, Massachusetts Institute of Technology

Mushotzky, R. F., \& Scharf, C. S. 1997, ApJ 482, 13

Oke, J.B. 1990, AJ, 99, 1621

Oke, J. B., Cohen, J. G., Carr, M., Cromer, J., Dingizian, A., Harris, F. H., Labrecque, S., Lucinio, R., Schaal, W., Epps, H., \& Miller, J. 1995, PASP, 107, 307

Ricker, P. M. 1998, ApJ 496, 670

Roettiger, K., Burns, J. O., \& Loken, C. 1996, ApJ, 473, 651

Tyson, J. A., Kochanski, G. P., \& Dell'Antonio, I. P. 1998 ApJ, 498 L107 
Table 1. Galaxy redshifts, relative to Abell $697 \mathrm{cD}$ at $\mathrm{z}=0.2824$

\begin{tabular}{crr}
\hline \hline Galaxy ID & $c z\left(\mathrm{~km} \mathrm{~s}^{-1}\right)$ & $\sigma(c z)$ \\
\hline $\mathrm{cD}$ & 0 & 0 \\
1 & 714 & 120 \\
3 & 278 & 70 \\
4 & 25 & 161 \\
6 & -2044 & 321 \\
7 & 758 & 30 \\
11 & 14 & 314 \\
14 & -2735 & 304 \\
20 & -779 & 155 \\
21 & -358 & 284 \\
\hline
\end{tabular}




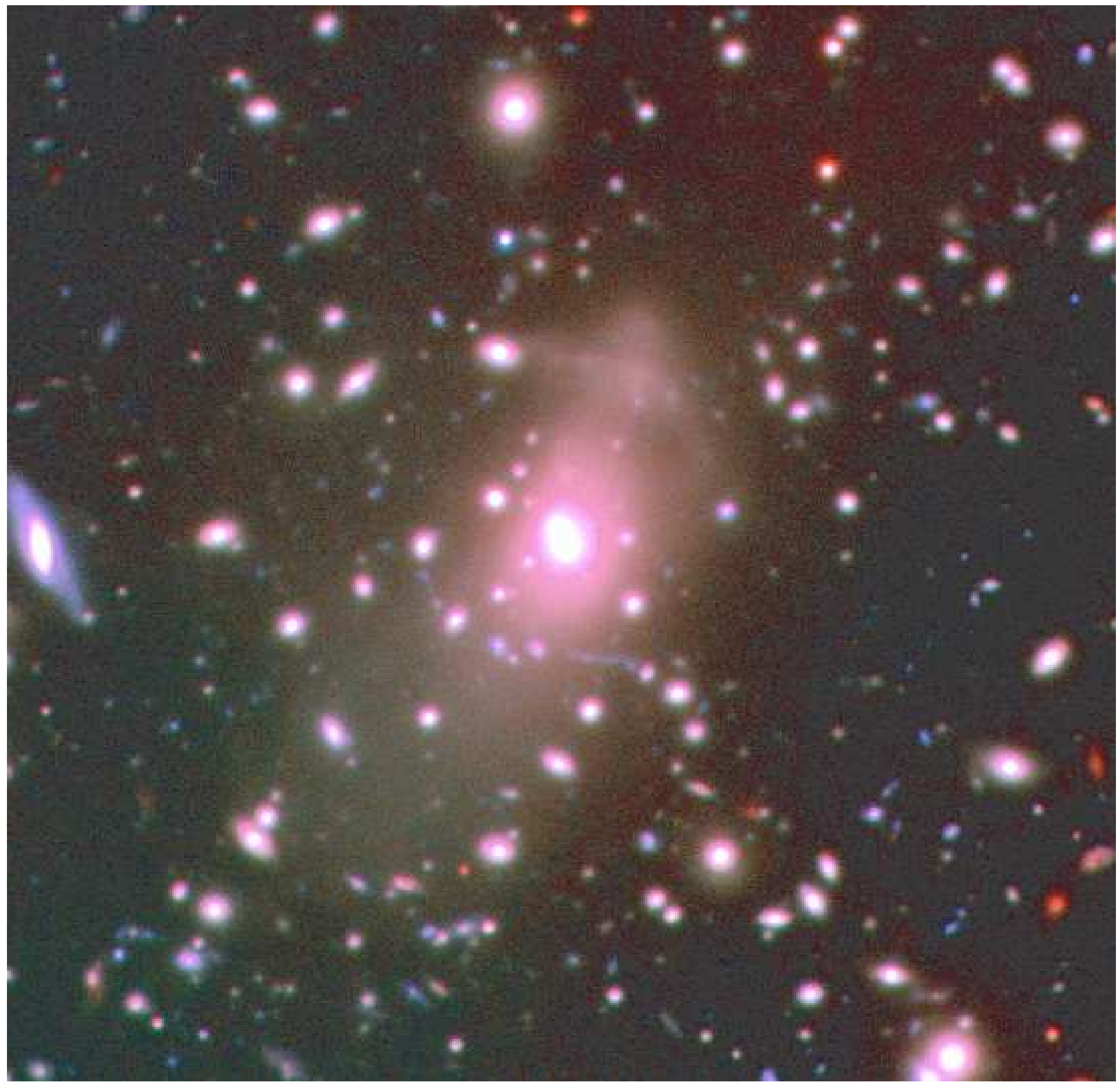

Fig. 1.- A false-color Keck/LRIS image of the central 120" of Abell 697. Blue, green, red correspond to the V, R, I band images, respectively. North is up and east is to the left. The lensed arc is visible to the south of the cluster, and the extended red source to the northwest. Exposure times were 3000 seconds in each band, and reaches a surface brightness limit of $28.2 \mathrm{mag} \operatorname{arcsec}^{-2}$ in $\mathrm{V}$. 


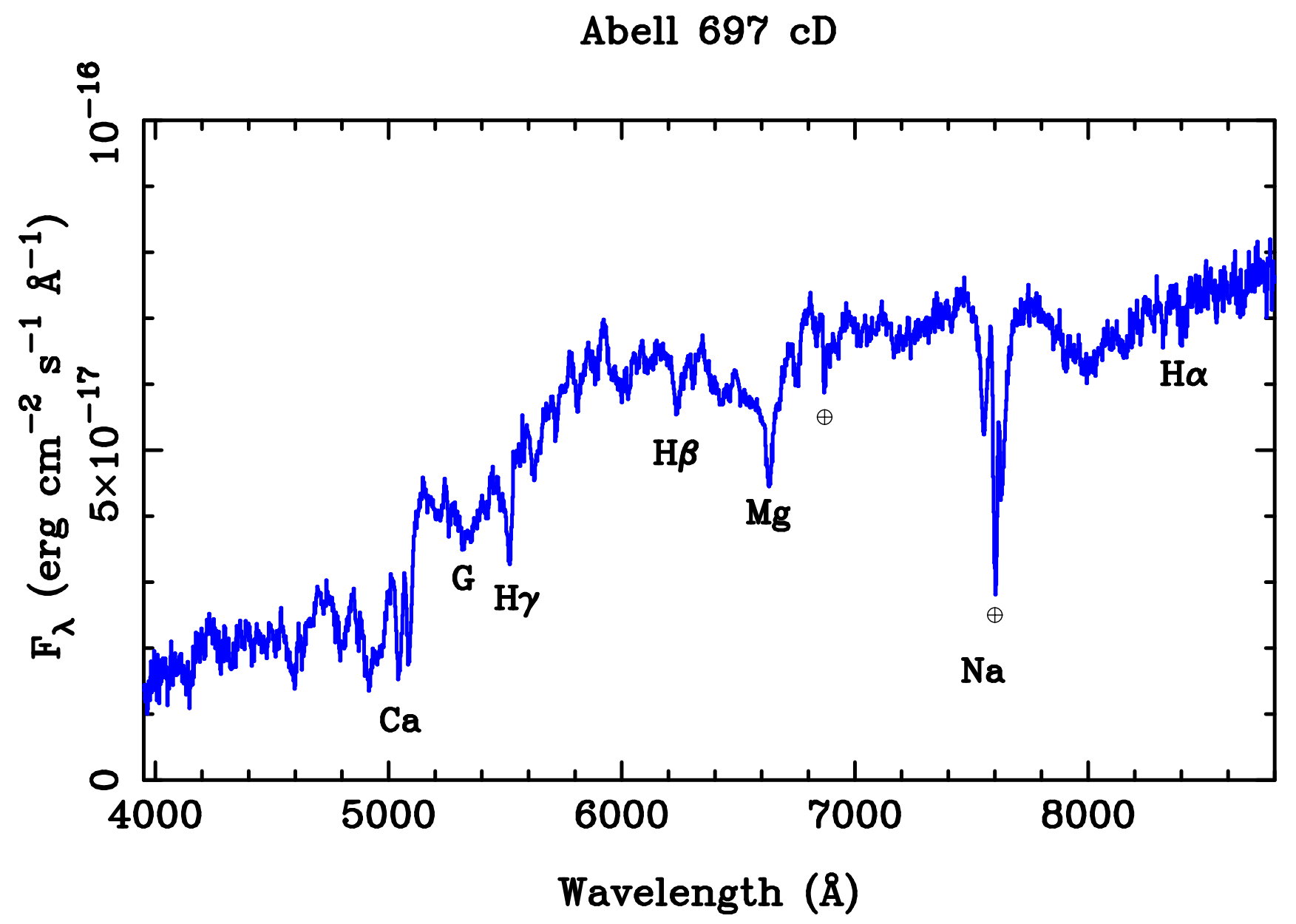

Fig. 2.- A spectrum of the Abell $697 \mathrm{cD}$ galaxy core, obtained with Keck/LRIS using a 1".0 slit. Telluric $\mathrm{O}_{2}$ A- and B-band absorption features are marked. No significant $\mathrm{H} \alpha$ emission is detected. 


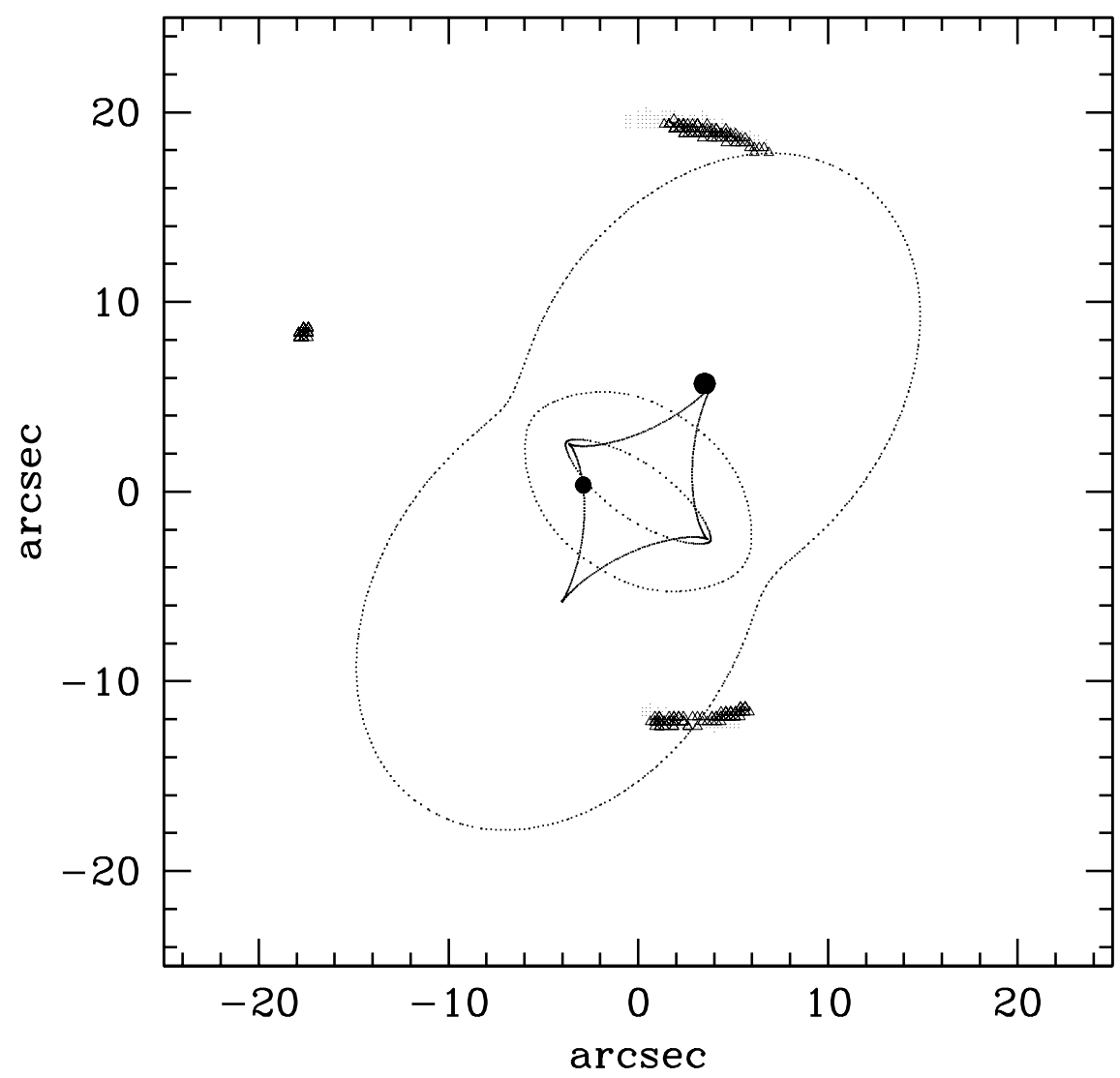

Fig. 3.- A plausible model for the Abell 697 arc candidates. The filled circle centered at $(-2.9,0.35)$ indicates the source position (assuming $\left.z_{s}=1.4\right)$ for the lower arc. The predicted counter image is at about $(-17,8)$. The inner two contours are the caustics and the outer two are the critical curves. The filled circle centered at $(3.5,5.7)$ labels the source position (with $z_{s}=1.2$ ) for the upper arc candidate. 